\title{
Adaptabilidade de Objetos de Aprendizagem usando Calibragem e Sequenciamento Adaptativo de Exercícios
}

\author{
Title: Adaptability of Learning Objects using Calibration and Adaptive Sequencing of \\ Exercises
}

\author{
Rômulo César Silva \\ UNIOESTE-campus Foz do Iguaçu \\ romulocesarsilva@gmail.com
}

\author{
Ana Carla Borille \\ UFPR \\ carlaborille@gmail.com
}

Bruno Filla Camargo

$U F P R$

brunofilla_camargo@hotmail.com

\author{
Alexandre Ibrahim Direne \\ UFPR \\ alexd@inf.ufpr.br \\ Paulo Ricardo B. Guimarães \\ UFPR \\ guimaraes.prb@gmail.com
}

\author{
Diego Marczal \\ UTFPR-campus Guarapuava \\ dmarczal@gmail.com
}

\author{
Angelo da Silva Cabral \\ $U F P R$ \\ angeloscabral@gmail.com
}

\section{Resumo}

Este trabalho aborda questões teóricas e de implementação de um arcabouço para a construção e execução de Objetos de Aprendizagem (OAs), em que as tarefas de resolução de problemas são ordenadas de acordo com o emparelhamento de 2 parâmetros calculados de maneira automática, formalmente definidos por expressões algébricas:(1) nível de expertise do aluno e (2) dificuldade de solução de um problema. O nível de habilidade é calculado automaticamente, expresso por um rating semelhante aos usados em jogos. O cálculo da dificuldade de solução é baseado em erros e acertos de estudantes ao lidar com o problema. As fórmulas algébricas desenvolvidas foram validadas mediante um estudo empírico realizado a partir de dados coletados de alunos reais. Também foi realizada uma avaliação experimental da aprendizagem utilizando um OA construído com o arcabouço para o domínio de logaritmos, aplicado a quatro turmas do ensino médio de uma escola pública, e os respectivos resultados são apresentados.

Palavras-Chave: Rating; Calibragem de Exercícios; Objetos de Aprendizagem.

\begin{abstract}
The work approaches theoretical and implementation issues of a framework for creating and executing Learning Objects (LOS) where problem-solving tasks are ordered according to the matching of two parameters, both calculated automatically: (1) student skill level and (2) problem solution difficulty. They are formally defined as algebraic expressions. The definition of skill level is achieved through a rating-based measure that resembles the ones of game mastery scales, while the solution difficulty is based on mistakes and successes of learners to deal with the problem. An empirical study based on existing students data demonstrated the suitability of the formulas. Besides, the motivational aspects of learning are considered in depth. In this sense, it is important to propose activities according to the student's level of expertise, which is achieved through presenting students with exercises that are compatible with the difficulty degree of their cognitive skills. Also, the results of an experiment conducted with four highschool classes using the framework for the domain of logarithmic properties are presented.
\end{abstract}

Keywords: Rating; Calibration of Exercises; Learning Objects.

Cite as: Silva, R.C.S., Direne, A.I., Marczal, D., Borille, A.C., Guimarães, P.R.B., Cabral, A.S. \& Camargo, B.F. (2018). Adaptability of Learning Objects using Calibration and Adaptive Sequence of Exercises (Adaptabilidade de Objetos de Aprendizagem usando Calibragem e Sequenciamento Adaptativo de Exercícios). Brazilian Journal of Computers in Education (Revista Brasileira de Informática na Educação - RBIE), 26(1), 70-90. DOI: 10.5753/RBIE.2018.26.01.70 


\section{Introdução}

A pesquisa sobre o desenvolvimento e uso de softwares educativos se diversificou bastante ao longo dos anos, contemplando diferentes abordagens e objetivos, não necessariamente excludentes, tais como: Objetos de Aprendizagem (OAs), Sistemas de e-learning, Sistemas Tutores Inteligentes (STIs) e Jogos Educacionais.

Enquanto OAs são auto-contidos em termos de conteúdo e têm como característica a busca pela reusabilidade/interoperabilidade (Wiley, 2000), os STIs procuram formas de se adaptar às necessidades individuais do estudante (Conati, 2009). Na abordagem de jogos educacionais procura-se ensinar ou reforçar determinado assunto exercitando alguma habilidade, valendo-se de características comuns a jogos como motivação e interação (Battistella, Wangenheim \& Fernandes, 2008; Savi \& Ulbricht, 2008).

De acordo com Anderson (2004) uma das motivações das pesquisas em STIs são as evidências que mostram que o tutor humano particular é muito eficaz. As decisões didáticas, ao invés de serem codificadas de maneira fixa, podem ser derivadas das interações de regras especializadas ou estruturas de conhecimento similar para representar a perícia pedagógica do sistema (Wenger, 1987; Woolf, 2009), sendo que representações explícitas de conhecimento pedagógico criam o potencial para sistemas se adaptarem e melhorarem suas estratégias ao longo do tempo, e para componentes serem reusados em outros domínios.

Nwana (1990) aponta duas razões principais porque os pesquisadores se interessam por STIs:

- necessidades de pesquisa: entender mais sobre os processos que contribuem para a interação educacional. Desde que STI está na interseção das principais disciplinas envolvidas, ele provê uma excelente base de teste para as várias teorias da Psicologia Cognitiva, IA e teorias educacionais;

- necessidades práticas: razões econômicas e sociais, em virtude da possibilidade de tutoria um para um.

Vanlehn (2006) observa que embora os STIs difiram bastante em domínios, interfaces de usuário, estruturas de software e bases de conhecimento, seus comportamentos na realidade são bastante similares, podendo ser descritos como tendo 2 laços (loops): um loop externo, executado uma vez para cada tarefa, no qual uma tarefa geralmente consiste em resolver um problema complexo de vários passos; e um loop interno, executado uma vez para cada passo tomado pelo estudante na construção da solução de uma tarefa. No loop interno, a competência do estudante pode ser avaliada e o modelo do estudante atualizado, que é usado pelo loop externo na escolha da próxima tarefa apropriada ao estudante.

Além disso, considerando os aspectos motivacionais em aprendizagem, é importante que o STI seja capaz de sugerir atividades adequadas ao nível da expertise do estudante. Isso porque a apresentação de exercícios com grau de dificuldade muito abaixo do nível cognitivo do aprendiz pode causar entediamento enquanto exercícios muito acima podem gerar desmotivação (Ravi, 2013). Ambas as situações podem gerar o abandono da atividade proposta.

$\mathrm{Na}$ literatura, nos trabalhos encontrados sobre sequenciamento automático de atividades, a granularidade do sequenciamento é no nível de objetos de aprendizagem (OAs) ou a validação do método de sequenciamento proposto foi feita apenas com estudantes simulados. Neste estudo é proposta uma técnica de sequenciamento para a granularidade do nível de exercícios, tanto em STIs como em OAs.

O sequenciamento automático de exercícios levando em conta o nível da expertise do aluno é relevante pelas seguintes razões: 
- permite maior grau de adaptabilidade dos STIs e OAs, principalmente no que se refere à adaptação do grau de dificuldade à capacidade do estudante;

- permite a construção de ferramentas de autoria com maior usabilidade, através da eliminação da necessidade do autor especificar as ordenações possíveis dos exercícios;

- diminui as horas de autoria necessárias à construção de STIs, reduzindo assim os seus custos.

Além disso, a correção automática dos exercícios aliada ao sequenciamento adequado pode contribuir na ampliação do potencial de avaliação formativa de ambientes de aprendizagem baseados em computador.

\section{Resenha Literária}

A pesquisa e desenvolvimento de Sistemas Tutores Inteligentes (STIs) tem procurado unir técnicas de IA, Psicologia Cognitiva e teorias de aprendizagem educacional com o objetivo de criar sistemas educacionais capazes de saber o que ensina, para quem ensina e como ensina (Nwana, 1990). Tradicionalmente, um STI é definido como sendo composto de 4 módulos (Wenger, 1987):

- Especialista (perito): cobre os fatos e regras do domínio particular a ser ensinado.

- Estudante (aprendiz): representa dinamicamente o conhecimento e a capacidade do estudante.

- Tutor (pedagógico): decide como o conteúdo deve ser apresentado ao estudante (tática pedagógica ou didática), adaptando-se à medida que o estudante interage com o sistema.

- Interface: é o componente de comunicação que controla a interação entre o estudante e o sistema.

\subsection{Modelagem do Estudante}

O modelo do estudante é considerado o componente central de um STI. Idealmente, deve conter tanto quanto possível todo o conhecimento sobre os estados cognitivo e afetivo do estudante e sua evolução à medida que o processo de aprendizagem avança. Geralmente, o modelo do estudante é visto como um modelo dinâmico que implementa várias funções. Wenger (1987) atribuiu 3 funções principais ao modelo do estudante:

- coletar dados implícitos e explícitos do estudante;

- usar esses dados coletados para criar a representação do conhecimento do estudante e do processo de aprendizagem;

- realizar algum tipo de diagnóstico levando em conta os dados coletados, seja do estado do conhecimento do estudante ou em termos de selecionar estratégias pedagógicas mais favoráveis para apresentação da informação de domínio subsequente para o estudante.

Em muitas visões da pesquisa de STIs, como na de Self (1990), a criação de modelos precisos do aprendiz permite atribuir ao estudante atividades práticas baseadas em avaliações de seu conhecimento pessoal. Além disso, modelos de estudante têm se tornado um elemento chave em STIs, apoiando o desenvolvimento de ajuda individualizada e detecção de comportamento desviado da tarefa proposta (Baker, Goldstein \& Heffernan, 2010). As abordagens mais recentes de comportamento deslocado do esperado são influenciadas pelo comportamento de outros estudantes. Nesse sentido, uma amostragem maior de dados dos alunos pode prover melhores avaliações automáticas de um aprendiz específico.

A construção de modelos do estudante pode ser realizada através de métodos de engenharia do conhecimento tal como entrevistas estruturadas ou esquemas automatizados como aprendizagem de máquina. As primeiras são muito subjetivas e dependem da natureza da codificação dedutiva da expertise do domínio. Geralmente, elas tomam bastante tempo e são 
suscetíveis a omissões de importante componentes do conhecimento. As técnicas automatizadas apresentam o desafio de interpretar os modelos gerados por algum método de natureza indutiva. Seguindo ambas abordagens, existem várias técnicas mencionadas na literatura para a construção de modelos do estudante, especialmente regras de produção, redes bayesianas, métodos fuzzy, ontologias, aprendizagem de máquina e mineração de dados (Nkambou, Bourdeau \& Mizoguchi, 2010).

Outro aspecto geralmente levado em conta na construção de modelos do estudante é se apenas uma ou múltiplas habilidades serão consideradas. Alguns modelos propostos são baseados na Teoria da Resposta ao Item (TRI), um modelo clássico em Psicometria que assume que o sucesso em todo item de um teste é determinado por uma única habilidade, representada por $\theta$, referida como traço latente. E além disso, há 2 parâmetros: discriminação $\left(a_{i}\right)$ e dificuldade do item $\left(b_{i}\right)$, usados para calcular o sucesso em um item conforme a Equação 1 (Desmarais \& Baker, 2011):

$$
P\left(X_{i} \mid \theta\right)=\frac{1}{1+e^{a_{i}\left(\theta-b_{i}\right)}}
$$

No caso de múltiplas habilidades, uma questão relevante é se as habilidades são consideradas independentemente ou se elas podem afetar umas às outras. Geralmente, o tratamento de múltiplas habilidades aumenta consideravelmente o esforço de autoria em STIs.

Cen, Koedinger and Junker (2008) e Gong, Beck and Herffernan (2010) propõem a utilização de modelos conjuntivos que assumem que o estudante deve dominar todas as habilidades requeridas em uma tarefa para realizá-la corretamente. Assim, seguindo a teoria de probabilidade clássica, a estimativa da probabilidade de realização correta da tarefa é feita pela multiplicação das probabilidades de que o estudante domine as habilidades requeridas.

$\mathrm{Xu}$ and Mostow (2011) criaram um modelo de aquisição de habilidades denominado LRDBN em que as probabilidades de transição entre estados de conhecimento sucessivos são calculadas usando regressão logística em todas as sub-habilidades. A regressão logística é uma técnica estatística que permite estabelecer relação entre uma variável resposta do tipo categórica e variáveis explicativas contínuas e/ou binárias. A utilização de regressão logística para modelagem de múltiplas habilidades visa evitar a subestimativa da probabilidade de acerto que ocorre geralmente em abordagens conjuntivas apresentadas anteriormente.

\subsection{Personalização em STIs}

Um dos desafios na construção de STIs é torná-los capazes de se adequar às necessidades individuais de aprendizagem do estudante. Assim, a personalização pode ir desde simples preferências de interface até a detecção de estados emocionais do estudante, podendo afetar a estruturação de todos os módulos do STI.

Uma das formas mais comuns de personalização em STIs é o emprego de suportes (scaffolding do inglês) adaptativos. Segedy and Biwas (2013) propuseram uma taxonomia para suportes adaptativos em sistemas de aprendizagem baseados em computador chamada SuggestAssert-Modify. Suportes do tipo sugestão proveem informação aos estudantes com o propósito de engajá-los em um comportamento específico. Suportes de asserção comunicam uma informação como sendo verdadeira que deve ser integrada com o seu atual entendimento. E suportes do tipo modificação altera aspectos da própria tarefa de aprendizagem.

No contexto da aprendizagem, é relevante considerar os diferentes níveis de conhecimento prévio e expertise dos estudantes, bem como suas habilidades cognitivas, estilos de aprendizagem e motivação, que em conjunto traduzem suas necessidades individuais. Nesse sentido, técnicas usadas em sistemas de recomendação também têm sido empregadas em STIs e em sistemas de e-learning para fins de adaptação às necessidades individuais do estudante 
(Feng, 2009; Cen, 2008). Existe um mapeamento similar entre modelagem do estudante em STIs e sistemas de recomendação onde estudante, tarefa (materiais), e performance (nota, pontuação) se torna usuário, item e rating, respectivamente (Thai-Nghe, 2011).

Um exemplo de uso de técnicas de sistemas de recomendação é o sistema tutor de programação Protus (Klašnja-Milićević, Vesin, Ivanocić, \& Budimac, 2011), que faz uso de ontologias do domínio assim como de regras de sintaxe para personalizar dinamicamente o material de apoio ao aprendiz. O sistema divide o processo de adaptação em 3 etapas. Inicialmente os estudantes são agrupados em clusters utilizando-se técnicas de mineração de dados de acordo com seu estilo de aprendizagem. Depois, padrões comportamentais são descobertos para cada estudante usando um algoritmo de mineração de $\log s$ Web. Em seguida, uma lista de recomendação é criada de acordo com as necessidades detectadas do estudante.

Segal, Katzir, Gal, Shani, and Shapira (2014) propõem um algoritmo denominado EduRank para personalizar conteúdo educacional para estudantes em sistemas e-learning, que combina algoritmos de filtragem colaborativa com a Teoria da Escolha Social (List, 2013). O algoritmo constrói um ranking de dificuldade sobre questões para um estudante destino agregando o ranking de estudantes similares, medido pelos aspectos diferentes de suas performances em questões comuns passadas, tais como série, número de tentativas e tempo gasto na solução de questões.

Outra funcionalidade que pode contribuir para a personalização em STIs é a capacidade de predição de performance do estudante. Especificamente, a previsão da performance tem por objetivo saber como os estudantes aprendem (em geral ou estritamente), o quão rapidamente ou lentamente se adaptam a novos problemas, ou ainda inferir os requisitos de conhecimento para solucionar os problemas, obtidos diretamente dos dados da performance do estudante. Além disso, eventualmente o sistema tenta descobrir se os estudantes realizariam as tarefas (problemas, exercícios) corretamente com algum nível de certeza, quando interagindo com os sistemas tutores (Thai-Nghe, 2011). Outro benefício da previsão de performance é permitir que os instrutores possam auxiliar o estudante provendo feedbacks antecipadamente.

Desarkar and Sarkar (2012) propõem um algoritmo de predição de rating chamado PrefNMF-RP, que considera os ratings relativos dados pelos usuários para diferentes pares de itens. $\mathrm{O}$ algoritmo modela os usuários e itens usando um framework de fatoração de matriz. $\mathrm{O}$ modelo de usuários e itens aprendido é usado primeiro para prever a utilidade personalizada de um item para um usuário. Essa utilidade é então convertida para um valor de rating válido dentro de uma escala pré-definida empregando uma classificação personalizada. $O$ algoritmo apresentou melhor performance que diferentes métodos baseados em filtragem colaborativa e fatoração de matriz.

Outra forma de personalização é através do uso de exercícios parametrizados que podem ser instanciados várias vezes com dados diferentes, sendo úteis em situações em que se deseja certificar que o estudante assimilou determinado conceito, solicitando o mesmo tipo de exercício ou suas variações mais de uma vez, porém com dados diferentes. Rioja, Santos, Pardo, and Kloos (2003) desenvolveram um STI baseado em exercícios parametrizados e hierarquicamente interconectados. O sequenciamento dos exercícios é representado através de um grafo criado por tutor humano tal que as arestas representam transições entre exercícios e são rotuladas com expressões booleanas contendo condições sobre a nota do estudante e número de tentativas. Embora o uso de exercícios parametrizados permita instanciação automática, o grafo de sequenciamento entre eles com as restrições de transições deve ser configurado por um especialista do domínio.

McNamara, Jackson, and Graesser (2009) discutem uma área adicional de pesquisa chamada Tutoria Inteligente e Jogos (ITaG - Intelligent Tutoring and Games do inglês), que 
incorpora princípios de jogos em STIs e vice-versa. Essa área de pesquisa seria uma fusão dos princípios de aprendizagem e motivação para criar jogos aprimorados com princípios de aprendizagem, e STIs aprimorados com princípios de jogos. Alguns exemplos de funcionalidades baseadas em jogos são competição, pontuação ou níveis e dificuldade de tarefas. É esperado que essas e outras funcionalidades, ao serem incluídas em STIs, possam aumentar os fatores motivacionais do estudante.

\subsection{Avaliação do Aprendiz}

Ferguson, Woolf, and Barto (2006) apontam que não é possível observar o domínio de uma habilidade ou perícia do aluno diretamente, sendo este conhecimento normalmente inferido a partir das respostas dadas por ele a problemas que envolvam a utilização dessas habilidades. Assim, a aferição e desenvolvimento da expertise do estudante é geralmente feita através da resolução de exercícios que requerem um conjunto de habilidades avaliadas. Isso é feito tanto no sistema educacional de sala de aula convencional quanto em sistemas que fazem uso de computadores e aplicam técnicas de Inteligência Artificial como os STIs.

Um erro do estudante pode basicamente ser usado para duas ações: simplesmente avaliar o estudante ou detectar equívocos conceituais visando uma prática pedagógica mais efetiva. Nessa última perspectiva, recentemente tem crescido o interesse pelo uso direto de erros como fonte de material didático, como por exemplo a técnica de exemplos errôneos. Essa técnica consiste de uma descrição de como solucionar um problema no qual um ou mais passos estão incorretos, sendo pedido ao estudante para encontrá-lo(s), explicar ou corrigir o(s) erro(s) visando fazê-lo aprender mais profundamente sobre o conteúdo do domínio e desenvolver habilidades metacognitivas (Isotani et al., 2011).

Existem diferentes abordagens para avaliação do aluno, seja em ambientes e-learning ou STIs. As avaliações podem ser automáticas, parcialmente automáticas ou ainda feitas por um especialista ou tutor humano.

Pinkwart, and Loll (2009) apresentaram um estudo comparativo entre três abordagens de avaliação da qualidade das soluções dos estudantes, sendo que duas fazem uso de revisões por pares: a qualidade da solução de uma tarefa de um estudante é determinada heuristicamente por avaliações de outros estudantes. Na primeira abordagem, quando um estudante trabalha em uma tarefa e provê uma solução, depois é solicitado a ele avaliar algumas soluções alternativas. Uma avaliação terá um peso maior que outras quando o estudante que a faz tem um rating de qualidade mais alto quando comparado a outros. A segunda abordagem assume que um estudante que consegue classificar corretamente a qualidade de soluções alternativas, também está apto a prover uma solução de maior qualidade. Enquanto a primeira faz um uso clássico de revisão por pares com um valor base fixo para aquelas soluções que não foram avaliadas ainda, a segunda substitui este valor base por um rating calculado dinamicamente. A terceira abordagem usa somente a fórmula de rating base. O processo de colaboração entre os usuários pode ser classificado como uma aplicação de Web Semântica Social, que tem o potencial de diminuir a sobrecarga dos tutores e prover a possibilidade dos estudantes treinarem suas capacidades de criticidade.

Guzmán and Conejo (2015) propõem um modelo de avaliação baseado na TRI para medir tanto o nível de conhecimento quanto os conceitos equivocados do estudante através de testes. $\mathrm{O}$ modelo se baseia na hipótese de que certas escolhas incorretas em questões de teste podem ser usadas para inferir os conceitos equivocados dos estudantes. Após a sessão de testes, o modelo do estudante é atualizado com informações a respeito do seu nível de conhecimento e também com dados sobre os conceitos equivocados assumidos por ele. $\mathrm{O}$ modelo de avaliação é tal que o relacionamento entre questões e conceitos, questões e conceitos equivocados é representado por curvas características da TRI. 
Sales, Barroso and Soares (2011) propõem um modelo de avaliação formativa para automatizar o acompanhamento qualitativo/quantitativo em ambientes virtuais de aprendizagem. $\mathrm{O}$ modelo faz uso de vetores na forma bidimensional e equações trigonométricas, em que os vetores-aprendizagem contém as avaliações em cada uma das atividades à distância do aluno tais como fóruns de discussão, tarefas, wikis, chats e também notas das atividades presenciais. A projeção horizontal do vetor expressa a nota naquela atividade e sua positividade enquanto a projeção vertical relaciona-se à negatividade do seu desempenho.

\subsection{Calibragem de Tarefas}

Um aspecto importante na elaboração de tarefas para serem realizadas pelos estudantes é a sua calibragem, ou seja, a determinação do seu nível de dificuldade. Do mesmo modo que um tutor humano, é desejável que sistemas de aprendizagem por computador sejam capazes de aferir o grau de dificuldades das tarefas que propõem.

Ravi and Sosnovsky (2013) propõem um método de calibragem para dificuldade de solução em STIs baseado na aplicação de técnicas de mineração de dados no $\log$ de interação do estudante com o sistema. Usando o método bayesiano clássico Knowledge Tracing (KT) (Corbett \& Anderson, 1994), a probabilidade que um estudante tenha adquirido uma habilidade é calculada com base na sequência de tentativas de exercícios para os quais as soluções envolvem um dado conceito. Os eventos no $\log$ são agrupados por exercícios e classificados de acordo com a habilidade do estudante. Os dados gerados são usados para se encaixarem na curva sigmoide da TRI (Baker, 2001) para conectar estudantes diferentes usando o algoritmo de clustering padrão $k$-means.

Papoušek, and Pelánek (2015) pesquisaram a relação entre o engajamento do estudante e a dificuldade da tarefa no ensino de Geografia, e propuseram um mecanismo para o ajuste dinâmico da dificuldade, em que cada item é avaliado por 3 funções de pontuação, cada uma de acordo com um critério diferente, tal que o item com a soma mais alta é usado como candidato para ser perguntado. $\mathrm{O}$ aspecto da dificuldade é levado em conta no modelo do estudante, que usa uma combinação do sistema de rating Elo do jogo de xadrez e PFA. Cada item tem uma probabilidade estimada $P_{\text {est }}$ que um estudante particular irá responder corretamente. A primeira função de pontuação depende da distância entre $P_{\text {est }}$ e a taxa de sucesso $P_{\text {target }}$. A segunda função, baseada no tempo gasto desde a última pergunta, serve para penalizar os itens. A terceira função induz o sistema a pedir questões sobre novos itens. A pontuação final é dada pela soma ponderada das pontuações individuais, sendo os pesos configurados manualmente a partir das experiências de uso do sistema.

Günel, and Asliyan (2009) propõem um modelo para calcular dificuldade de questões em STIs usando a seguinte equação diferencial:

$$
y(t)=\frac{y_{0}}{\left.y_{0}+\left(1-y_{0}\right) \cdot e^{-k t} \quad\right)^{(2}}
$$

onde $y_{0}=0.5$ e $k=\frac{\beta-\alpha}{\alpha+\beta}$, sendo $\alpha$ e $\beta$ o número de vezes que a questão foi respondida corretamente, e com falha, respectivamente. Os autores pontuam a necessidade de testar a fórmula com alunos reais para verificar sua efetividade. 


\subsection{Sequenciamento de Tarefas}

O sequenciamento de tarefas refere-se à ordem em que as diferentes atividades de aprendizagem (exercícios, leitura, interação com outros alunos) devem ser propostas aos alunos. É eminentemente uma atribuição do módulo tutor, mas tem estreita relação com a calibragem de tarefas e com a avaliação da performance do aprendiz.

Clement, Roy and Oudeyer (2014) propõem dois algoritmos para modelo tutor de STI. O primeiro, chamado RiARiT (Right Activity at Right Time do inglês), é baseado em técnicas usadas em problemas de alocação sequencial de recursos, conhecidas como multi-arm bandits na gíria americana da língua inglesa (Bubeck \& Cesa-Bianchi, 2012), tal que cada atividade envolve diferentes capacidades, referidas como componentes de conhecimento (do inglês Knowledge Components (KCs). O modelo do estudante é uma generalização do modelo bayesiano KT na qual o nível de competência do estudante é representado por um número real no intervalo [0..1]. Também há uma tabela que associa para cada atividade o nível de competência mínimo requerido de cada componente de conhecimento para que se obtenha sucesso. Quando o estudante tem sucesso na atividade, assume-se que seu nível de competência está acima do mínimo, caso contrário ele estará abaixo. Além disso, uma recompensa representando o progresso de aprendizagem é definida pela diferença entre o nível de competência do estudante o nível de competência mínimo requerido. $\mathrm{O}$ algoritmo inclui um conjunto de filtros que atuam como especialistas que monitoram o quanto de recompensa cada atividade está dando, e seleciona as atividades de ensino proporcionalmente ao progresso esperado de aprendizagem.

O segundo algoritmo, ZPDES (Zone of Proximal Development and Empirical Success) (Clement et al., 2014) é uma versão modificada de RiARiT, em que o cálculo da recompensa é mudado para remover a dependência do nível de competência estimado do estudante. A recompensa torna-se uma medida de como a taxa de sucesso está aumentando, provendo uma escolha mais preditiva de atividades. Uma limitação do estudo é a falta de validação com estudantes reais, sem fazer suposições a respeito de suas taxas de aprendizagem e níveis de compreensão.

Champaign and Cohen (2010) propõem um algoritmo para sequenciamento de conteúdo que seleciona o objeto de aprendizagem (OA) apropriado a ser apresentado a um estudante, baseado em experiências de aprendizagem prévias de usuários similares. A granulação de sequenciamento ocorre no nível do OA, não de exercícios ou questões. Uma limitação do trabalho é que o algoritmo foi validado usando somente estudantes simulados.

Schatten and Schmidt-Thieme (2014) apresentam o Vygotski Policy Sequencer (VPS), baseado no conceito de Zona de Desenvolvimento Proximal (ZDP) idealizado por Vygotski. Nessa abordagem, a matriz de fatoração, que é um método para predição de rating de usuário, é combinada com uma política de sequenciamento, visando selecionar a cada passo o conteúdo de acordo com o escore estimado. O VPS é constituído de tal forma que a definição explícita de dificuldade não é necessária. Os autores também mostram como usar estudantes simulados para testar as sequências antes de serem aplicadas a estudantes reais.

Pimentel and Direne (1998) apresentam uma ferramenta de autoria denominada SEQUENCE para medidas cognitivas usadas no ensino de conceitos visuais. A ferramenta permite que o autor do STI utilize a carga cognitiva de diagnóstico dos exemplares para a estruturação da sequência de sessões de ensino. Em um primeiro nível, o autor define o formato de uma sessão de ensino: número de imagens que a compõe, quais imagens, sua ordem de apresentação e parâmetros para controlar a apresentação das imagens. Em um outro nível, é possível descrever os valores que cada imagem de uma classe de anomalia possui, permitindo diferenciá-las qualitativa e quantitativamente. A carga cognitiva de uma imagem é calculada 
levando-se em conta parâmetros do domínio tais como: frequência, saliência, confiabilidade, visão tridimensional, diagnóstico diferencial, sinônimos técnicos, e também pesos definidos por um especialista. Um estudo de caso demonstrou que a sequência definida por um especialista e a definida pela ferramenta de autoria foram relativamente semelhantes, demonstrando assim sua eficiência.

\section{Conceitos da Solução}

Geralmente professores detectam equívocos conceituais dos alunos durante a realização de testes e exercícios. Dependendo de como a solução dada diverge da resposta correta ou solução esperada, dois estudantes que erraram a mesma questão podem ser pontuados diferentemente para aquela questão específica.

Considerando que todas questões estejam bem formuladas, outra característica que pode ser usada para compor a nota é o grau de dificuldade da questão, podendo ser medido pelo número de estudantes que a saltaram ou erraram. Assim, um estudante que responde corretamente uma questão que muitos erraram, provavelmente tem mais capacidade no assunto avaliado que os demais e sua nota deve refletir isso. Inversamente, um estudante que erra uma questão que vários outros acertam, possivelmente tem menos capacidade no tópico avaliado.

Este trabalho propõe uma fórmula para avaliação da perícia do aprendiz, um método para calibragem de exercícios e um algoritmo associado para sequenciamento em OAs, visando apresentar ao estudante exercícios de grau de dificuldade compatível com sua performance dinâmica.

\subsection{Cálculo Automático de Rating}

A fórmula para classificar a perícia do aluno foi inspirada em sistemas de rating de jogos adversaristas como xadrez. O rating é um número real em um intervalo [minRank, maxRank] tal que é muito improvável um jogador (ou aluno) atingir os extremos do intervalo.

As seguintes diretrizes foram adotadas na elaboração da fórmulas de rating do aluno e grau de dificuldade das questões:

- a cada questão é atribuído um grau de dificuldade no intervalo [0..10];

- similarmente, cada estudante tem seu rating no intervalo [1..10] para expressar sua perícia no assunto em questão;

- quanto mais fácil uma questão, maior a probabilidade que os estudantes a respondam corretamente. Nesse caso, o rating de um estudante terá um pequeno aumento caso acerte a questão ou maior diminuição no caso de erro;

- estudantes que têm sucesso na primeira tentativa de solução de uma questão são pontuados com uma nota mais alta no seu rating quando comparados com outros que precisaram de várias tentativas;

- questões saltadas são consideradas erradas.

Este estudo propõe a Equação 3 para avaliar iterativamente a habilidade de um estudante, e considera os acertos, os erros e o número de tentativas, tanto do próprio aluno quanto dos demais, como se houvesse implicitamente uma competição entre todos os alunos da turma. 


$$
\begin{aligned}
R_{J}^{(q)}=R_{J}^{(q-1)} & +A \times K_{1} \times \alpha\left(10-\frac{9 T_{J}^{(q)}}{T_{m e d}^{(q)}}\right)-E \times K_{2} \\
& \times \beta\left(10 \frac{T_{J}^{(q)}}{T_{m e d}^{(q)}}\right)
\end{aligned}
$$

onde:

- $\quad R_{J}^{(q)}$ : rating do estudante $J$ após responder a questão $q$;

- $A=1$ e $E=0$ se o estudante acertou $q$, caso contrário $A=0$ e $E=1$;

- $T_{J}^{(q)}$ : número de tentativas sem sucesso do estudante $J$ ao responder a questão $q$;

- $T_{\text {med }}^{(q)}$ : mediana do número de tentativas erradas da questão $q$ durante a sessão de exercícios;

- $\quad \alpha=\frac{1}{N_{a}^{q}}$ : ponderação do incremento de rating, onde $N_{a}^{q}$ é o número de estudantes que tiveram sucesso em responder a questão $q$;

- $\beta=\frac{1}{N_{e}^{q}}$ : ponderação do decremento de $\left\{\right.$ lit rating\}; onde $N_{e}^{q}$ é o número de estudantes que erraram a questão $q$;

- $K_{1}$ e $K_{2}$ são fatores multiplicadores de incremento e decremento de rating, respectivamente, calculados de acordo $\operatorname{com} R_{J}^{(q-1)}$ tal que $1 \leq R_{J}^{(q-1)} \leq 10, K_{1}=$ $1-\frac{R_{J}^{(q-1)}}{10} \$$ e $K_{2}=\frac{R_{J}^{(q-1)}-1}{10}$

Além disso, não existe limite para o número de tentativas. Entretanto, se há mais de 10 (dez) tentativas, 10 é considerado para propósitos de cálculo. Os fatores $K_{1}$ e $K_{2}$ evitam que o resultado da Equação 3 atinja os extremos do intervalo [1..10]. O rating inicial $R_{J}^{(0)}$ é 5.5 (ponto médio do intervalo de rating) para todos estudantes inicialmente, quando não se tem qualquer informação a seu respeito.

\subsection{Grau de Dificuldade}

Em uma primeira abordagem, o grau de dificuldade da questão $q$ é definido através dos detalhes da Equação 4 e seus parâmetros que se seguem:

$$
D^{(q)}=\frac{N_{e}^{(q)}+N_{s}^{(q)}}{N_{e}^{(q)}+N_{a}^{(q)}+N_{s}^{(q)}} \times 10
$$

onde:

- $\quad D^{(q)}$ : grau de dificuldade da questão $q$ após uma sessão de exercícios;

- $N_{e}^{(q)}$ : número de estudantes que erraram $q$;

- $N_{a}^{(q)}$ : número de estudantes que acertaram $q$;

- $N_{s}^{(q)}$ : número de estudantes que saltaram $q$.

Em outra abordagem, usando somente o número de tentativas e considerando que o estudante geralmente tenta até conseguir obter a resposta correta, o grau de dificuldade de uma questão $q$ pode ser definido pela Equação 5 e seus parâmetros que seguem:

$$
D^{(q)}=\frac{\sum_{0}^{n} T_{J}^{(q)}}{N_{e}^{(q)}+N_{a}^{(q)}}
$$


onde:

- $\quad D^{(q)}$ : grau de dificuldade da questão $q$ após uma sessão de exercícios;

- $T_{J}^{(q)}$ : número de tentativas do estudante $J$ na questão $q$. Se o número de tentativas é maior que 10 , então 10 é considerado como $T_{J}^{(q)}$;

- $N_{e}^{(q)}$ : número de estudantes que erraram $q$;

- $N_{a}^{(q)}$ : número de estudantes que acertaram $q$.

Nesse caso, quanto mais difícil a questão, maior a probabilidade de que os estudantes precisem de mais tentativas para chegar à resposta correta. Além disso, o número de estudantes que saltaram $q$ não é considerado.

\subsection{Algoritmo para Sequenciamento Adaptativo de Exercícios}

Um aspecto importante em STIs é como os exercícios devem ser sequenciados após serem calibrados tal que se encaixem com o nível de perícia do estudante. No início, o sistema não tem qualquer informação a respeito do estudante. Portanto, uma maneira natural é colocá-los em ordem crescente de dificuldade. Entretanto, existem muitas questões em aberto relacionadas ao número de exercícios que devem ser apresentados, e quantas vezes.

Neste trabalho é proposto um algoritmo para sequenciamento de exercícios em ordem crescente de dificuldade, combinado com um mecanismo similar à interpolação numérica, que segue as diretrizes:

- uma sequência mínima de exercícios é definida para ser apresentada ao estudante;

- a sequência mínima sempre começa com o exercício mais fácil e termina com o mais difícil;

- os exercícios de dificuldade intermediária na sequência mínima são distribuídos uniformemente entre o mais fácil e o mais difícil tal que o número de exercícios que compõem a sequência $\left(N_{\text {seq_min }}\right)$ é dado pela Equação 6:

$$
N_{\text {seq_min }}=\left\lfloor\frac{n}{s}\right\rfloor+1+\left\lfloor\frac{n \bmod s}{2}\right\rfloor
$$

onde $n$ é o total de exercícios, e $s$ é stepsize, que refere-se ao número de exercícios que podem ser saltados quando o estudante acerta o exercício;

- o número de tentativas que um estudante tem para resolver uma questão é limitado à média de tentativas obtidas durante a fase de calibragem;

- quando o número de tentativas excede o limite estabelecido, o próximo exercício a ser apresentado ao estudante é o que se encontra no ponto médio de dificuldade entre o exercício atual e o último respondido corretamente.

Por exemplo, considere um OA com 30 exercícios em ordem crescente de dificuldade $\left[e_{1}, e_{2}, \ldots, e_{30}\right]$ e stepsize $=4$. A sequência mínima de exercícios será composta pelos exercícios $\left.<e_{1}, e_{5}, e_{9}, e_{13}, e_{17}, e_{21}, e_{25}, e_{29}, e_{30}\right\rangle$, e os exercícios serão apresentados ao estudante considerando inicialmente esta ordem. Porém, por exemplo, se o estudante excede o número de tentativas estabelecido para $e_{9}$, então $e_{7}$ (ponto médio entre $e_{5}$ e $e_{9}$ ) é apresentado. Ao contrário do que ocorre durante a fase de calibragem dos exercícios, o(a) estudante não pode saltar exercícios. Além disso, se ele/ela erra continuadamente, a apresentação dos exercícios passa a ser sequencial. O stepsize pode ser configurado pelo autor do $\mathrm{OA}$, devendo ser tal que o número de exercícios na sequência mínima tenha pelo menos $25 \%$ do total de exercícios. 
Na Figura 1 é apresentada uma árvore de sequenciamento de exercícios considerando stepsize $=4$. Nós-filhos à direita representam o próximo exercício quando o estudante respondeu corretamente ao exercício correspondente ao nó-pai. Enquanto o estudante erra um exercício $e_{t}$, o mesmo é apresentado novamente até o limite de tentativas. Após isso, o exercício representado pelo nó-filho à esquerda é exibido.

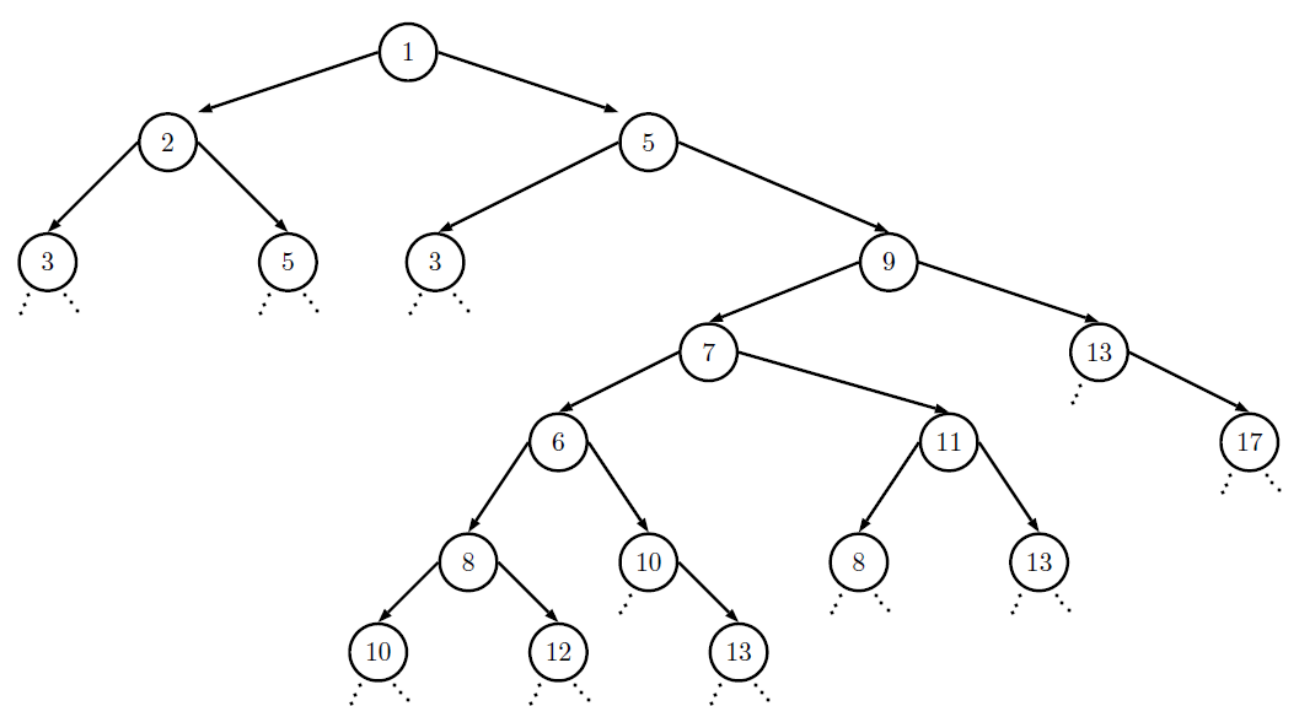

Figura 1: árvore de sequenciamento para stepsize $=4$.

O algoritmo de sequenciamento mostrado no Pseucódigo 1 tem as seguintes funções auxiliares:

- ultimo_exercicio_resolvido: retorna o último exercício feito pelo estudante;

- proximo_exercicio_nao_resolvido_seq_minima: retorna o próximo exercício da sequência mínima ainda não resolvido;

- primeiro_exercicio_nao_resolvido: retorna o primeiro exercício ainda não apresentado após o último resolvido na sequência mínima;

- ultimo_ex_correto: retorna o último exercício respondido corretamente da sequência mínima;

- ultimo_exercicio_correto_anterior: retorna o último exercício respondido corretamente anterior ao exercício-argumento passado;

- primeiro_exercicio_nao_resolvido_a_partir: retorna o primeiro exercício não resolvido a partir do ponto médio dos argumentos informados;

- Correto: retorna verdadeiro se o estudante resolveu corretamente o exercício;

- Media: retorna a média de tentativas obtidas durante a fase de calibragem.

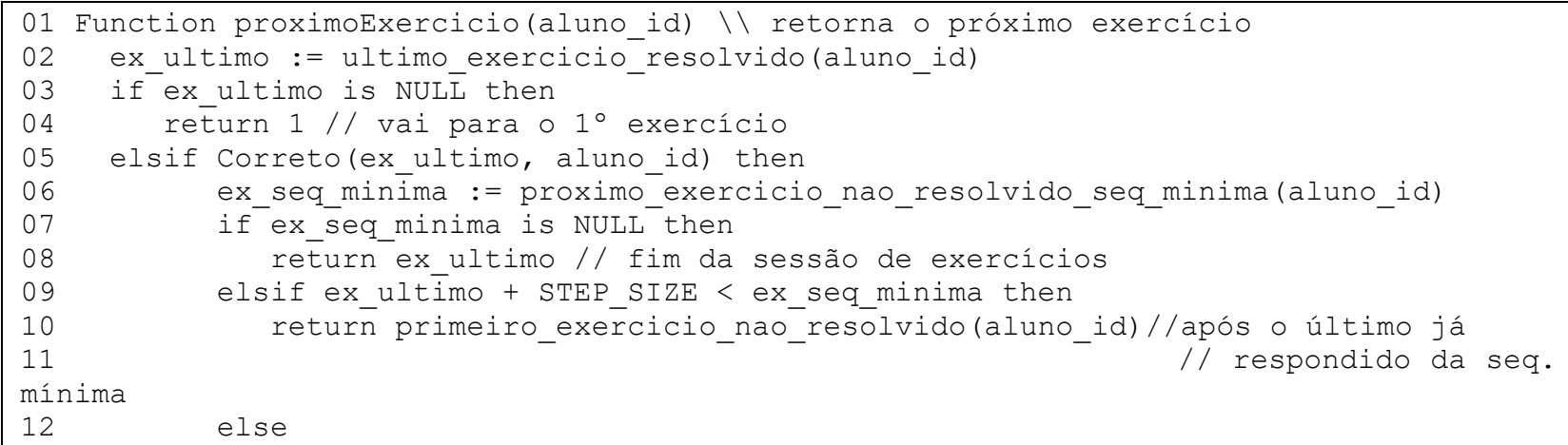




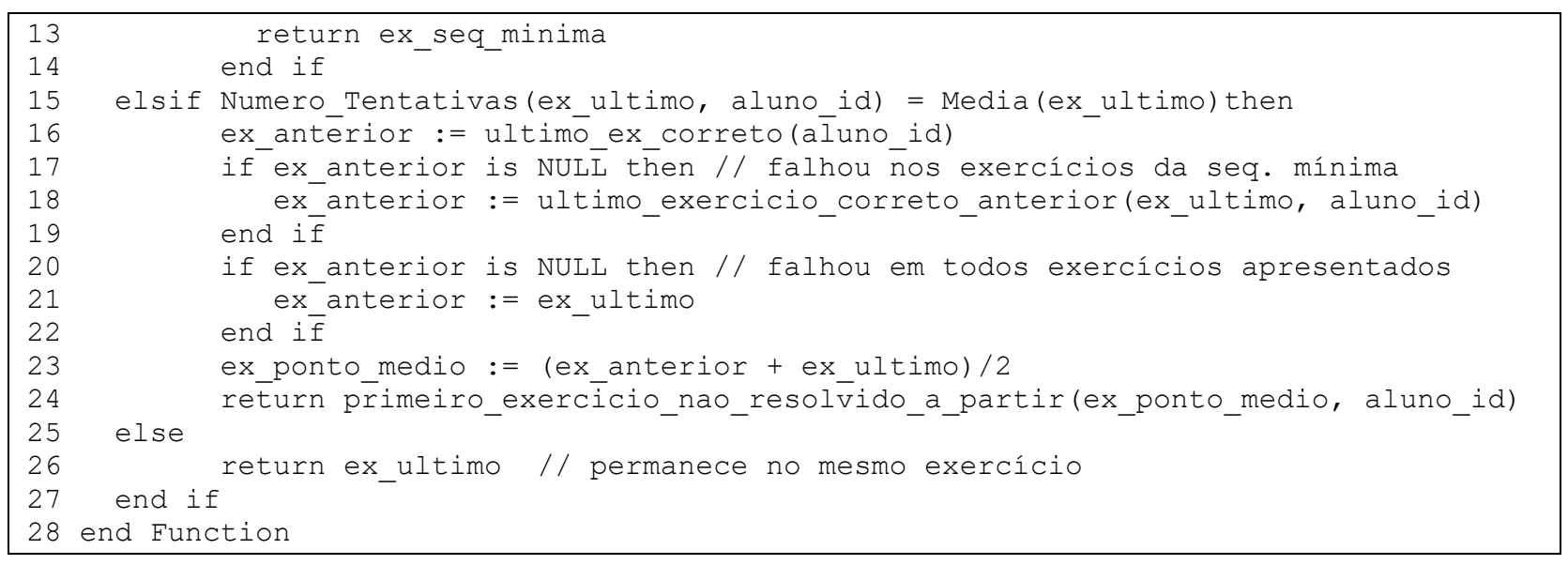

Pseudocódigo 1: Função para Retornar o Próximo Exercício.

\subsection{Estudo Empírico}

Antes de fazer a avaliação experimental do algoritmo de sequenciamento, foi realizado um estudo empírico para validar o comportamento das fórmulas de rating e grau de dificuldade. Para tal, foram utilizados dados anteriormente coletados de alunos reais por 2 (dois) OAs diferentes: um sobre Progressão Geométrica em Fractais, aplicado em uma turma de 34 alunos do $1^{\circ}$ ano de Engenharia, e outro OA sobre Logaritmos, aplicado em uma turma de 21 alunos do $1^{\mathrm{o}}$ ano do ensino médio do curso Técnico de Edificações.

Para a validação da fórmula de rating foi feita sua aplicação iterativa para cada aluno seguindo a ordem de apresentação das questões no OA, enquanto para o grau de dificuldade, foram empregadas diretamente as fórmulas e, tendo ambas obtidas o comportamento esperado.

Também foi verificado que $T_{\max }^{(q)}$ (número máximo de tentativas na questão $q$ ) pode ser usada no lugar de $T_{\text {med }}^{(q)}$ (mediana do número de tentativas da questão $q$ ), desde que se estabeleça um valor limite, visando descartar valores discrepantes gerados por alunos que respondem várias vezes ao mesmo exercício de maneira descompromissada. A implementação do cálculo de $T_{\max }^{(q)}$ tem complexidade de tempo $O(n)$ enquanto a implementação clássica de $T_{\text {med }}^{(q)}$, que requer ordenação, tem complexidade $O(n \log n)$.

\section{O software ADAPTFARMA}

ADAPTFARMA é uma versão modificada de FARMA (Ferramenta de Autoria para a Remediação de erros com Mobilidade na Aprendizagem) (Marczal, 2014; Marczal \& Direne, 2012) uma ferramenta de autoria para construção de OAs de conceitos matemáticos. Do mesmo modo que em FARMA, em ADAPTFARMA um OA consiste de uma sequência de exercícios após uma introdução. A introdução é a parte teórica de um OA onde conceitos são definidos através de texto, imagens, áudios e vídeos. Os exercícios contêm a essência prática dos conceitos, com os quais o aprendiz irá adquirir perícia através de tarefas de resolução de problemas. Adicionalmente, ADAPTFARMA tem integrado em seu arcabouço a implementação do cálculo de rating dos alunos, o grau de dificuldades das questões e o algoritmo de sequenciamento adaptativo de exercícios apresentados na seção 3.

Para construir uma introdução e seus exercícios correspondentes, ADAPTFARMA oferece uma interface WYSIWYG (What You See Is What You Get). Em relação aos exercícios, a ferramenta é bastante genérica, permitindo ao professor definir o número de questões relacionadas a cada exercício. Esta técnica é baseada na teoria ACT (Adaptive Control of 
Thought) (Ritter, Anderson, Koedinger \& Corbett, 2007). Para cada questão, o professor-autor deve indicar uma solução de referência, que é a resposta correta da questão. ADAPTFARMA permite que expressões algébricas e aritméticas sejam colocadas como solução de referência. Sob o modo estudante, a ferramenta lida automaticamente com equivalência entre a resposta do estudante e a solução de referência.

Além disso, ADAPTFARMA permite ao professor-autor definir regras de remediação e mensagens para cada questão dos exercícios, aplicadas quando o estudante comete um erro. Para tal, é necessário definir o número de tentativas sem sucesso após o qual a mensagem de feedback deve ser exibida. Isto mostra o quanto é simples proporcionar feedback imediato em ADAPTFARMA.

A capacidade de retroagir ao contexto exato do erro cometido pelo estudante é uma funcionalidade diferenciada de ADAPTFARMA (Marczal \& Direne, 2011), quando comparada a ferramentas similares, pois dá oportunidade ao professor de identificar os passos equivocados feitos pelo estudante para então lidar apropriadamente com as causas do erro. Além disso, ADAPTFARMA permite ao professor ver uma interação completa do estudante com a ferramenta em ordem cronológica, na forma de uma timeline. Da mesma maneira, os aprendizes também podem retroagir ao contexto exato de qualquer uma das suas respostas corretas ou erradas, visando refletir sobre seus próprios passos da solução.

ADAPTFARMA é arquiteturalmente dividida em 3 módulos principais: autoria, interação e monitoramento. $\mathrm{O}$ módulo de autoria provê as funcionalidades para construção de OAs. O módulo de interação é a interface entre o aprendiz e o OA criado pelo professor, efetuando a junção das introduções, exercícios, questões e remediação de erros, para executar o OA como uma sequência de páginas.

O módulo de monitoramento permite que o professor faça a avaliação formativa da aprendizagem individual do aluno através da retroação a erros, em que o professor pode ter acesso ao contexto exato da ocorrência de erro do aluno, além de relatórios disponíveis mostrando o progresso de cada aluno da turma. Esse módulo é composto pelos seguintes submódulos:

- Indexador de Respostas: salva todas respostas submetidas pelo estudante, sejam erradas ou corretas;

- Modelo do Estudante: a informação da interação de cada usuário com o OA particular, tais como:

- Progresso do Aprendiz, que analisa as respostas dos estudantes e informa em porcentagem, quanto cada estudante falta para completar o OA;

- O timeline do Aprendiz, onde é possível ver todas interações em ordem cronológica;

○ Visão do Estudante do OA, onde o professor tem exatamente a mesma visão do estudante;

- Motor de Busca, no qual o professor, bem como estudantes podem pesquisar por erros;

- Relatório sobre Interação, onde é possível ver de maneira tabulada a interação dos aprendizes com o OA, agrupada por: nome do estudante, exercícios, questão, resposta correta ou incorreta; e o número de tentativas da questão.

- Retroação a Respostas, em que o indexador de respostas recupera dados sobre o aprendiz e permite o professor retornar para o contexto exato da resposta de um estudante específico. 


\section{Avaliação Experimental}

Esta seção descreve um experimento realizado e os respectivos resultados obtidos, cujo objetivo foi comparar o efeito na aprendizagem da estratégia de sequenciamento implementada pelo algoritmo mostrado no Pseudocódigo 1 da seção 3.3, com outras estratégias de sequencimento. $\mathrm{O}$ experimento foi realizado com a aplicação de um mesmo objeto aprendizagem em turmas de estudantes do Ensino Médio, porém usando estratégias de sequenciamento diferentes.

\subsection{Sujeitos}

O experimento foi realizado com 4 (quatro) turmas de estudantes do Ensino Médio de uma escola pública, sendo 2 turmas do curso Técnico em Química Industrial (turmas A e B), e 2 turmas do curso Técnico em Meio Ambiente (turmas C e D). A idade dos estudantes é entre 15 e 17 anos. A Tabela 1 apresenta a quantidade de alunos que participaram em cada etapa do experimento, sendo a última coluna o número de alunos que participaram de todas as etapas.

Tabela 1: Quantidade de Alunos Participantes por Etapa.

\begin{tabular}{|c|c|c|c|c|c|}
\hline Turma & Total de Alunos & Pré-teste & ADAPTFARMA & Pós-teste & Válidos \\
\hline A & 33 & 31 & 30 & 32 & 29 \\
\hline B & 35 & 32 & 33 & 34 & 30 \\
\hline C & 33 & 33 & 30 & 32 & 30 \\
\hline D & 35 & 32 & 32 & 35 & 30 \\
\hline
\end{tabular}

Foram retirados da análise do experimento os alunos que não realizaram o pré-teste ou pós-teste ou que não utilizaram a ferramenta, totalizando 119 participantes válidos.

\subsection{Material}

Foi criado um OA sobre logaritmos na ADAPTFARMA com 10 páginas conceituais e 30 exercícios, elaborado e aplicado pelo professor de Matemática das 4 turmas. O professor também foi o responsável pela elaboração e aplicação do pré-teste e pós-teste aplicados nas 4 turmas, valendo 1,5 pontos cada, sendo a pontuação usada para compor a nota oficial do registro acadêmico. Os conteúdos de ambos pré-teste e pós-teste versavam sobre o mesmo assunto abordado no OA (logaritmos), sendo compostos de exercícios similares aos do OA.

\subsection{Metodologia}

O mesmo pré-teste foi aplicado às 4 turmas. Posteriormente, o OA criado sobre Logaritmos foi aplicado, sendo que a ferramenta ADAPTFARMA estava disponível online e cada turma teve 2 dias alternados com 2 horas-aulas de 50 min em laboratório de informática, equipado com computadores com acesso à Internet para utilizá-la.

Para cada turma foi usada uma estratégia de sequenciamento diferente, distribuídas da seguinte maneira:

- turma A: método de sequenciamento randômico (MSR);

- turma B: método de sequenciamento definido pelo professor (MSP);

- turma C: método de sequenciamento em ordem crescente de dificuldade (MSD), onde o grau de dificuldade foi calculado segundo a Equação 5 definida na Seção 3.2 usando os dados obtidos da fase de calibragem da turma A;

- turma D: método de sequenciamento adaptativo (MSA), aplicando o algoritmo exibido no Pseudocódigo 1 da Seção 3.3.

As turmas A (sequenciamento do professor) e B (sequenciamento randômico) foram tratadas como grupo de controle do experimento. 
Na Tabela 2 é mostrada a correspondência das ordenações dos exercícios do OA obtidas para as estratégias cujo sequenciamento é fixo para todos os alunos, isto é, MSP, MSD e MSR. Assim, por exemplo: o $1^{\circ}$ exercício na ordem fixada pelo professor corresponde ao $2^{\circ}$ na ordem por grau de dificuldade e ao $7^{\circ}$ pela ordenação randômica.

Para os métodos MSR, MSP e MSD não havia limite para o número de tentativas, enquanto para o MSA a média de tentativas da turma A foi utilizada.

\subsection{Resultados}

O teste Shapiro-Wilk foi aplicado às notas obtidas para checar normalidade. Como somente os dados do MSD passaram no teste de normalidade $(p$-valor $=0,0827)$, então o Teste T pareado foi aplicado a essa turma, enquanto para as outras três, foi aplicado o teste Wilcoxon para avaliar individualmente cada método de sequenciamento. Os p-valores resultantes estão exibidos na Tabela 3, mostrando que com exceção de MSD, todos os métodos tiveram um aumento significativo nos escores entre o pré e o pós-teste.

Tabela 2: Correspondência das Ordenações dos Exercícios.

\begin{tabular}{|c|c|c|}
\hline MSP & MSD & MSR \\
\hline $1^{\mathrm{o}}$ & $2^{\circ}$ & $7^{\mathrm{o}}$ \\
\hline $2^{\circ}$ & $9^{\circ}$ & $25^{\circ}$ \\
\hline $3^{\circ}$ & $1^{\circ}$ & $13^{\circ}$ \\
\hline $4^{\circ}$ & $23^{\circ}$ & $12^{\circ}$ \\
\hline $5^{\circ}$ & $20^{\circ}$ & $5^{\circ}$ \\
\hline $6^{\circ}$ & $21^{\circ}$ & $19^{\circ}$ \\
\hline $7^{0}$ & $14^{\circ}$ & $28^{\circ}$ \\
\hline $8^{\circ}$ & $13^{\circ}$ & $23^{\circ}$ \\
\hline $9^{\circ}$ & $12^{\circ}$ & $16^{\circ}$ \\
\hline $10^{\circ}$ & $18^{\circ}$ & $11^{\circ}$ \\
\hline $11^{\circ}$ & $15^{\circ}$ & $14^{\circ}$ \\
\hline $12^{\circ}$ & $30^{\circ}$ & $2^{\circ}$ \\
\hline $13^{\circ}$ & $16^{\circ}$ & $18^{\circ}$ \\
\hline $14^{\circ}$ & $7^{\circ}$ & $4^{\circ}$ \\
\hline $15^{\circ}$ & $3^{\circ}$ & $17^{\circ}$ \\
\hline $16^{\circ}$ & $4^{0}$ & $29^{\circ}$ \\
\hline $17^{\circ}$ & $22^{\circ}$ & $8^{\circ}$ \\
\hline $18^{\circ}$ & $19^{\circ}$ & $21^{\circ}$ \\
\hline $19^{\circ}$ & $11^{\circ}$ & $10^{\circ}$ \\
\hline $20^{\circ}$ & $5^{\circ}$ & $24^{\circ}$ \\
\hline $21^{\circ}$ & $24^{\circ}$ & $3^{\circ}$ \\
\hline $22^{\circ}$ & $28^{\circ}$ & $6^{\circ}$ \\
\hline $23^{\circ}$ & $17^{\circ}$ & $26^{\circ}$ \\
\hline $24^{\circ}$ & $29^{\circ}$ & $9^{\circ}$ \\
\hline $25^{\circ}$ & $26^{\circ}$ & $15^{\circ}$ \\
\hline $26^{\circ}$ & $6^{\circ}$ & $30^{\circ}$ \\
\hline $27^{\circ}$ & $25^{\circ}$ & $27^{\circ}$ \\
\hline $28^{\circ}$ & $10^{\circ}$ & $22^{\circ}$ \\
\hline $29^{\circ}$ & $8^{\circ}$ & $20^{\circ}$ \\
\hline $30^{\circ}$ & $27^{\circ}$ & $1^{o}$ \\
\hline
\end{tabular}


Tabela 3: Avaliação Individual dos Métodos de Sequenciamento.

\begin{tabular}{|c|c|c|c|c|}
\hline $\begin{array}{c}\text { Tipo de } \\
\text { Sequenciamento }\end{array}$ & $\begin{array}{c}\text { Diferença Média } \\
\text { entre Notas }\end{array}$ & Shapiro-Wilk & Teste T Pareado & Wilcoxon \\
\hline MSR & 0,1759 & 0,0041 & - & 0,0007 \\
\hline MSP & 0,2033 & 0,0039 & - & $<0,0001$ \\
\hline MSD & 0,0233 & $\mathbf{0 , 0 8 2 7}$ & 0,5302 & - \\
\hline MSA & 0,1400 & 0,0428 & - & 0,0037 \\
\hline
\end{tabular}

O método ANOVA foi aplicado aos dados do pré-teste que exibiram normalidade, obtendo $p$-valor $=0,2539$, demonstrando não haver diferença significativa entre as 4 turmas. Para os dados do pós-teste e diferença média entre pré e pós-testes foi aplicado o teste Kruskal-Wallis, obtendo $p$-valor $=0,00579$ e $p$-valor $=0,03073$ respectivamente, demonstrando existir diferença significativa e sugerindo que MSR, MSP e MSA levam o estudante a obter um melhor desempenho que MSD. Os resultados estão mostrados na Tabela 4.

Além disso, o desempenho entre MSR, MSP e MSA foi similar. Surpreendentemente, MSR obteve a melhor performance enquanto MSD a pior. Isto contradiz um grande número de pesquisas referenciadas na literatura sobre práticas pedagógicas usando computadores (Self, 1990) ou por outro lado, para desenvolvimento de habilidades de solução de problemas. Algumas razões que podem explicar esse fenômeno são:

- o problema da ordenação de exercícios é uma questão relevante que deve ser explorada mais detalhadamente para permitir a verificação da influência tácita do conhecimento contido na organização textual do problema;

- a falta de diferença significativa entre MSR, MSP e MSA é também confirmada por evidências achadas em pesquisas passadas, como no experimento descrito em Major and Reichgelt (1992) e Mitrovic, Koedinger, and Martin (2003).

- o MSD pode ter se ligado a algum tópico relevante que causou um aumento da carga cognitiva, resultando em soluções de problemas que divergem das corretas;

- embora a maioria dos estudantes tenham participado do experimento, somente as notas do pré- e pós-teste foram levadas em conta na nota final constante no registro acadêmico do estudante.

Tabela 4: Comparação entre os Métodos de Sequenciamento.

\begin{tabular}{|c|c|c|c|c|}
\cline { 2 - 3 } \multicolumn{1}{c|}{} & \multicolumn{2}{c|}{ Pressupostos ANOVA } & \multicolumn{1}{c|}{} \\
\hline Comparação & Shapiro-Wilk & Bartlett & ANOVA & Kruskall-Wallis \\
\hline Pré-testes & $\mathbf{0 , 1 1 5 9}$ & $\mathbf{0 , 1 1 5 0}$ & $\mathbf{0 , 2 5 3 9}$ & - \\
\hline Pós-testes & 0,0011 & $\mathbf{0 , 7 6 4 6}$ & - & 0,0056 \\
\hline $\begin{array}{c}\text { Diferenças do Pós-teste } \\
\text { para o Pré-teste }\end{array}$ & 0,0062 & $\mathbf{0 , 7 5 6 6}$ & - & 0,0307 \\
\hline
\end{tabular}

\section{Conclusão e Trabalhos Futuros}

Geralmente a perícia do estudante é desenvolvida pela solução de exercícios que requerem um conjunto de habilidades avaliadas, inclusive em STIs. Este trabalho propôs um sistema de rating automático que pode ser usado como ferramenta adicional de avaliação dos estudantes. Dependendo do número de tentativas e o grau de dificuldade de uma questão, estudantes podem obter notas diferentes para a mesma questão. Além disso, duas fórmulas para o cálculo do grau de dificuldade de uma questão foram apresentadas.

$\mathrm{O}$ sistema de rating implicitamente estabelece um ranking entre os estudantes que pode auxiliar os professores identificarem quem necessita mais assistência. Além disso, ele permite que o professor obtenha um feedback sobre o grau de dificuldade das questões que elabora, e 
que essa informação possa ser agregada em repositórios de exercícios, habilitando futuramente a montagem automática de OAs.

Este estudo também propôs um algoritmo para sequenciamento de exercícios que usa o grau de dificuldade combinado com um mecanismo similar à interpolação numérica. $\mathrm{O}$ algoritmo foi implementado para compor o ambiente ADAPTFARMA, uma ferramenta de autoria web com interface WYSIWYG para criação e execução de OAs. O ambiente ADAPTFARMA está implementado de tal forma que é muito simples alterar a estratégia de sequenciamento de exercícios. Aproveitando dessa característica, foi realizado um experimento com quatro turmas do Ensino Médio de uma escola pública, para testar as seguintes estratégias de sequenciamento: MSR, MSP, MSD e MSA. Surpreendentemente somente MSD não obteve um aumento significativo nas notas dos alunos e MSR obteve a melhor performance, demonstrando que o problema da ordenação de exercícios é uma questão relevante, devendo ser pesquisada mais cuidadosamente.

O algoritmo implementado em MSA permite aumentar o grau de adaptabilidade de OAs desenvolvidos no ambiente ADAPTFARMA, pois o sequenciamento dos exercícios é feito automaticamente conforme o desempenho do estudante. Além disso, permite eliminar a necessidade do autor do OA precisar especificar as ordenações possíveis dos exercícios. O mesmo algoritmo poderia também ser aplicado em STIs, obtendo os mesmos benefícios e contribuindo assim para diminuir o número de horas necessárias a sua construção.

A correção automática de exercícios feita em ADAPTFARMA, aliada ao sequenciamento adequado pode contribuir na avaliação formativa de ambientes de aprendizagem baseados em computador, pois tanto o aluno quanto o professor têm feedback imediato sobre a correção dos exercícios realizados.

Os trabalhos futuros serão concentrados em adicionar funcionalidades ao ambiente ADAPTFARMA, que atuem de duas maneiras. Primeiro desenvolvendo uma abordagem mais profunda de adaptação ao usuário que inclua mais dimensões além da unificação entre a dificuldade do problema e a capacidade do estudante. Por exemplo, uma funcionalidade de geração de exercícios baseados em parâmetros definidos pelo professor. Segundo, relacionado à interface, ter mais modos de interação disponíveis para melhorar tarefas de colaboração para monitoramento do progresso da performance do aluno.

\section{Referências}

Anderson, J. R. (2004). Psicologia Cognitiva e suas Implicações Experimentais. LTC.

Baker, F. B. (2001). The Basics of Item Response Theory. University of Wiscosin: ERIC Clearinghouse on Assessment and Evaluation. [GS Search]

Baker, R. S. J. d., Goldstein, A. B., \& Heffernan, N. T. (2010). Detecting the Moment of Learning. LNCS, 6094(PART I), 25-34. doi: 10.1007/978-3-642-13388-6 7 [GS Search]

Battistella, P. E., von Wangenheim, C. G., \& Fernandes, J. M. (2014). Como jogos educacionais são desenvolvidos? Uma revisão sistemática da literatura. In WEI - XXII Worshop sobre Educação em Computação - CSBC 2014 (pp. 1445-1455). SBC. [GS Search]

Bubeck, S., \& Cesa-Bianchi, N. (2012). Regret Analysis of Stochastic and Nonstochastic Multiarmed Bandit Problems. Foundations and Trends ${ }^{\circledR}$ in Machine Learning, 5(1), 1-122. doi: $\underline{10.1561 / 2200000024}$ [GS Search]

Cen, H. (2008). Generalized Learning Factors Analysis: Improving Cognitive Models with Machine Learning. Carnegie Mellon University. 
Cen, H., Koedinger, K., \& Junker, B. (2008). Comparing Two IRT Models for Conjunctive Skills. In B. P. Woolf, E. Aimeur, R. Nkambou, \& S. Lajoie (Eds.), Proceedings of the the 9th International Conference of Intelligent Tutoring Systems (ITS 2008) (Vol. 5091, pp. 796-798). Springer Berlin Heidelberg. [GS Search]

Champaign, J., \& Cohen, R. (2010). A Model for Content Sequencing in Intelligent Tutorign Systems Based on the Ecological Approach and Its Validation Through Simulated Students. In Proceedings of the the 23rd Florida Artificial Intelligence Research Society Conference (FLAIRS 2010) (pp. 486-491). Association for the Advancement of Artificial Intelligence (AAAI). [GS Search]

Clement, B., Roy, D., \& Oudeyer, P.-Y. (2014). Online Optimization of Teaching Sequences with Multi-Armed Bandits. In J. Stamper, Z. Pardos, M. Mavrikis, \& B. M. McLaren (Eds.), Proceedings of the 7th International Conference on Educational Data Mining (pp. 269272). [GS Search]

Conati, C. (2009). Intelligent Tutoring Systems: New Challenges and Directions. In Proceedings of the 20th International Joint Conference on Artificial Intelligence (IJCAI'09) (pp. 2-7). San Francisco. [GS Search]

Corbett, A. T., \& Anderson, J. R. (1994). Knowledge tracing: Modeling the Acquisition of Procedural Knowledge. User Modeling and User-Adapted Interaction, 4(4), 253-278. doi:10.1007/BF01099821 [GS Search]

Desarkar, M. S., \& Sarkar, S. (2012). Rating prediction Using Preference Relations Based Matrix Factorization. In K. Y. Eelco Herder \& S. Weibelzahl (Eds.), Workshop and Poster Proceedings of the 20th Conference on User Modeling, Adaptation, and Personalization (UMAP2012) (Vol. 872). http://ceur-ws.org/. [GS Search]

Desmarais, M. C., \& Baker, R. S. J. D. (2011). A review of recent advances in learner and skill modeling in intelligent learning environments. User Modeling and User-Adapted Interaction, 22(1-2), 9-38. doi:10.1007/s11257-011-9106-8

Feng, M. (2009). Towards Assessing Students' Fine Grained Knowledge: Using an Intelligent Tutor for Assessment. Worcester Polytechnic Institute. [GS Search]

Ferguson, K., Woolf, B., \& Barto, A. (2006). Improving Intelligent Tutoring Systems: Using Expectation Maximization To Learn Student Skill Levels, 1-22. [GS Search]

Gong, Y., Beck, J. E., \& Heffernan, N. T. (2010). Comparing Knowledge Tracing and Performance Factor Analysis by Using Multiple Model Fitting Procedures. LNCS, 6094(PART I), 35-44. [GS Search]

Günel, K., \& Asliyan, R. (2009). Determining Difficulty of Questions in Intelligent Tutoring Systems. In The Turkish Online Journal of Educational Technology - TOJET (Vol. 8). [GS Search]

Guzmán, E., \& Conejo, R. (2015). Measuring Misconceptions Through Item Response Theory. In C. Conati, N. Heffernan, A. Mitrovic, \& M. F. Verdejo (Eds.), Proceedings of 17th International Conference on Artificial Intelligence in Education (AIED 2015) (Vol. 9112, pp. 608-611). Springer International Publishing Switzerland. doi: 10.1007/978-3-31919773-9 73 [GS Search]

Isotani, S., Adams, D., Mayer, R. E., Durkin, K., Rittle-Hohnson, B., \& McLaren, B. M. (2011). Can Erroneous Examples Help Middle-School Students Learn Decimals? In C. D. K. et al. (Ed.), European Conference on Technology Enhanced Learning (EC-TEL 2011) (Vol. 6964, pp. 181-195). Palermo: Springer Berlin Heidelberg. [GS Search] 
Klašnja-Milićević, A., Vesin, B., Ivanocić, M., \& Budimac, Z. (2011). E-learning Personalization based on Hybrid Recommednation Strategy and Learning Style Identification. Computers \& Education, 56(3), 885-899.

List, C. (2013). Social Choice Theory. In E. N. Zalta (Ed.), The Stanford Encyclopedia of Philosophy (Winter 2013). [GS Search]

Major, N., \& Reichgelt, H. (1992). COCA: A Shell for Intelligent Tutoring Systems. In Proc. of the International Conference on Intelligent Tutoring Systems (ITS92) (pp. 523-530). Springer.

Marczal, D. (2014). Farma: Uma Ferramenta de Autoria para Objetos de Aprendizagem de Conceitos Matemáticos. Universidade Federal do Paraná, UFPR. [GS Search]

Marczal, D., \& Direne, A. (2011). Um Arcabouço que Enfatiza a Retroação a Contextos de Erro na Solução de Problemas. Revista Brasileira de Informática na Educação, 19(1), 63-73. doi: $10.5753 /$ rbie.2011.19.01.63 [GS Search]

Marczal, D., \& Direne, A. (2012). FARMA: Uma ferramenta de autoria para objetos de aprendizagem de conceitos matemáticos. In Anais do Simpósio Brasileiro de Informática na Educação (Vol. 23).

McNamara, D. S., Jackson, G. T., \& Graesser, A. (2009). Intelligent Tutoring and Games (ITaG). In H. C. Lane, A. Ogan, \& V. Shute (Eds.), AIED 2009 Workshops Proceedings Volume 3: Intelligent Educational Games (pp. 1-10). University of Southern California.

Mitrovic, A. (2003). An Intelligent SQL Tutor on the Web. International Journal of Artificial Intelligence in Education, 13(3), 173-197. [GS Search]

Mitrovic, A., Koedinger, K. R., \& Martin, B. (2003). A Comparative Analysis of Cognitive Tutoring and Constraint-Based Modeling. In P. Brusilovsky, A. Corbett, \& F. Rosis (Eds.), User Modeling 2003, 9th International Conference (UM 2003) (pp. 313-322). JohnstownPA: Springer Berlin Heidelberg. [GS Search]

Nkambou, R., Bourdeau, J., \& Mizoguchi, R. (2010). Advances in Intelligent Tutoring Systems (Vol. 308). Springer. [GS Search]

Nwana, H. S. (1990). Intelligent Tutoring Systems: an overview. Artificial Intelligence Review, 4(4), 251-277. [GS Search]

Papoušek, J., \& Pelánek, R. (2015). Impact of Adaptive Educational System Behaviour on Student Motivation. In C. C. et al. (Ed.), 17th International Conference on Artificial Intelligence on Education (AIED 2015) (Vol. 9112, pp. 348-357). Madrid: Springer. [GS Search]

Pimentel, A. R., \& Direne, A. I. (1998). Medidas Cognitivas para o Ensino de Conceitos Visuais com Sistemas Tutores Inteligentes. In R. S. Wazlawick (Ed.), Revista Brasileira de Informática na Educação (Vol. 2, p. 17-24). [GS Search]

Pinkwart, N., \& Loll, F. (2009). Comparing Three Approaches to Assess the Quality of Students' Solutions. In D. Dicheva, R. Mizoguchi, \& N. Pinkwart (Eds.), AIED 2009 Workshops Proceedings Volume 2, SWEL'09: Ontologies and Social Semantic Web for Intelligent Educational Systems Intelligent Educational Games (pp. 81-85). [GS Search]

Ravi, G. A., \& Sosnovsky, S. (2013). Exercise difficulty Calibration Based on Student Log Mining. In F. Mödritscher, V. Luengo, E. L.-C. Law, \& U. Hoppe (Eds.), Proceedings of DAILE'13: Workshop on Data Analysis and Interpretation for Learning Environments. Villard-de-Lans (France). [GS Search] 
Rioja, R. M. G., Santos, S. G., Pardo, A., \& Kloos, C. D. (2003). A Parametric Exercise Based Tutoring System. In 33rd Annual Frontiers in Education, 2003 (FIE 2003) (Vol. 3, p. S1B_20-S1B_26). doi: 10.1109/FIE.2003.1265906 [GS Search]

Ritter, S., Anderson, J. R., Koedinger, K. R., \& Corbett, A. (2007). Cognitive Tutor: Applied Research in Mathematics Education. Psychonomic Bulletin \& Review, 14(2), 249-255. [GS Search]

Sales, G. L., Barroso, G. C., \& Soares, J. M. (2011). Um Indicador de Aprendizagem Não-linear para EaD online Fundamentado no Modelo de Avaliação Learning Vectors (LV). In Anais do XXII SBIE - XVII WIE (pp. 1713-1722). [GS Search]

Savi, R., \& Ulbricht, V. R. (2008). Jogos Digitais Educacionais: Benefícios e Desafios. RENOTE - Novas Tecnologias Na Educação, 6(2). [GS Search]

Schatten, C., \& Schmidt-Thieme, L. (2014). Adaptive Content Sequencing without Domain Information. 6th International Conference on Computer Based Education. [GS Search]

Segal, A., Katzir, Z., Gal, K., Shani, G., \& Shapira, B. (2014). EduRank: A Collaborative Filtering Approach to Personalization in E-learning. In P. Z. M. M. M. B. M. Stamper J. (Ed.), Proceedings of the 7th International Conference on Educational Data Mining (pp. 68-75). [GS Search]

Segedy, J. R., Loretz, K. M., \& Biswas, G. (2013). Suggest-Assert-Modify: A Taxonomy of Adaptive Scaffolds in Computer-Based Learning Environments. In G. Biswas, R. Azevendo, V. Shute, \& S. Bull (Eds.), AIED 2013 Workshops Proceedings Volume 2: Scaffolding in Open-Ended Learning Environments (OELEs) (pp. 73-80). [GS Search]

Self, J. (1990). Bypassing the Intractable Problem of Student Modelling, (41), 1-26.

Thai-Nghe, N. (2011). Predicting Student Performance in a Intelligent Tutoring System. Department of Computer Science, Information Systems and Machine learning Lab (ISMLL), University of Hildesheim, Germany.

Vanlehn, K. (2006). The Behavior of Tutoring Systems. International Journal of Artificial Intelligence, 16(3), 227-265. [GS Search]

Wenger, E. (1987). Artificial Intelligence and Tutoring Systems: Computational and Cognitive Approaches to the Communication of knowledge. Morgan Kaufmann.

Wiley, D. A. (2000). The Instructional Use of Learning Objects: Online Version. In D. A. Wiley (Ed.). http://www.reusability.org/read/ [GS Search]

Woolf, B. P. (2009). Building Intelligent Interactive Tutors: Student-centered Strategies for Revolutionizing e-Learning. San Francisco, CA, USA: Morgan Kaufmann Publishers Inc.

Xu, Y., \& Mostow, J. (2011). Using Logistic Regression to Trace Multiple Subskills in a Dynamic Bayes Net. In M. Pechenizkiy, T. Calders, C. Conati, S. Ventura, C. Romero, \& J. Stamper (Eds.), Proceedings of the the 4th International Conference on Educational Data Mining (EDM 2011) (pp. 241-245). Eindhoven, Holanda: International Educational Data Mining Society. [GS Search] 